\title{
DOPING PROPERTIES OF AMPHOTERIC C, Si, AND Ge IMPURITIES IN GaN AND AIN
}

\author{
P. Boguslawski ${ }^{a, b}$ AND J. BeRnhol $\mathrm{C}^{b}$ \\ ${ }^{a}$ Institute of Physics, Polish Academy of Sciences \\ Al. Lotników 32/46, 02-668 Warsaw, Poland \\ ${ }^{b}$ Department of Physics, North Carolina State University, Raleigh, NC 27695, USA
}

\begin{abstract}
Electronic structure of substitutional group-IV impurities $\mathrm{C}, \mathrm{Si}$, and $\mathrm{Ge}$ in hexagonal GaN and AlN were studied by quantum molecular dynamics. $\mathrm{C}_{\mathrm{N}}$ is a very shallow acceptor, and thus a promising $p$-type dopant. Both $\mathrm{Si}$ and Ge are excellent donors in GaN. However, in AlGaN alloys the DX configurations are stable for a sufficiently high $\mathrm{Al}$ content, which quenches the doping efficiency. Electronic structure of nearest-neighbor $X_{\text {cation }}-X_{N}$ pairs is also discussed.
\end{abstract}

PACS numbers: 71.55.-i

Current interest in wide band-gap nitrides is due to possible applications in blue/UV light-emitting diodes and lasers, and in high-temperature electronics $[1,2]$. To exploit fully the potential of these materials, understanding and control of doping needs to be achieved. In the present paper we study substitutional group-IV $\mathrm{X}=\mathrm{C}, \mathrm{Si}$, and Ge impurities in the hexagonal (wurtzite) GaN and AlN. These species are potentially important dopants (e.g., $\mathrm{Si}$ is frequently used as a $n$-type dopant of epitaxial $\mathrm{GaN}$ ). On the other hand, both $\mathrm{C}$ and Si may be unintentionally incorporated as contaminants during growth.

In general, a group-IV atom is likely to become a donor when incorporated on the cation site, and an acceptor on the anion site. This possibility of the amphoteric behavior critically depends on the conditions of growth. We.have recently shown [3] that $\mathrm{C}$ is preferentially incorporated on the $\mathrm{N}$ site under Ga-rich conditions of growth, while both Si and Ge occupy cation sites under N-rich conditions. However, under other conditions self-compensation, i.e., a simultaneous incorporation of the dopant on both cation and anion sublattice, is expected. For this reason we discuss here the electronic structure of $\mathrm{X}_{\text {cation }}, \mathrm{X}_{\mathrm{N}}$, and $\mathrm{X}_{\text {cation }}-\mathrm{X}_{\mathrm{N}}$ nearest-neighbor pairs. We also consider a possible transition of $X_{\text {cation }}$ from the substitutional to the DX-like configuration. Such a transition is commonly accompanied by a capture of electron on a stable (or metastable) state, which quenches the doping efficiency. Some of the results for $\mathrm{C}$ were published in [4]. The calculations were performed using quantum molecular dynamics [5]. Technical details were given in [6]. Soft pseudopotentials for $\mathrm{N}$ and $\mathrm{C}$ were used [7], while the pseudopotential of Ge was generated according to Ref. [8]. 
We first briefly discuss configurations of substitutional impurities and strain effects. A substitutional impurity in a wurtzite crystal has four nearest neighbors. One of them, located along the $c$-axis relative to the impurity (called here type-1 neighbor), is non-equivalent to the remaining three neighbors (called here type-2 neighbors). For the group-IV atoms considered here, this non-equivalence is small, since the bond lengths with type- 1 and type -2 neighbors are equal to within 1 per cent. In all cases, we find breathing mode distortions preserving the local hexagonal symmetry. The calculated changes in bond lengths are given in Table, together with the energy gain $E_{\text {rel }}$ due to the relaxation from the ideal substitutional configuration to the final one. As follows from Table, the inclusion of relaxation effects is necessary for a proper description of $\mathrm{C}_{\text {cation }}, \mathrm{Si}_{\mathrm{N}}$, and $\mathrm{Ge}_{\mathrm{N}}$, due to the large differences between the atomic radii of the impurity and host atoms. The most drastic case is that of $\mathrm{Ge}_{\mathrm{N}}$ in AlN: the lattice relaxation releases the elastic energy $\Delta E_{\text {rel }}$ of $7 \mathrm{eV}$, rises the acceptor level by $1.05 \mathrm{eV}$, and increases the Ge-N bonds by 17 per cent. Very similar results are obtained for AlN:Si. The calculated $\Delta E_{\text {rel }}$ are systematically greater for AlN than for $\mathrm{GaN}$, reflecting the higher stiffness of AlN.

\section{TABLE}

Effects of atomic relaxations around impurities. $\Delta b$ is the change of the bond length, $\Delta E_{\text {rel }}$ is the relaxation energy, $E_{\text {imp }}$ is the position of the impurity level, with e.m. denoting the effective-mass state, and $\Delta E_{\mathrm{imp}}$ is the relaxation-induced;shift of the impurity level.

\begin{tabular}{|c|c|c|c|c|}
\hline & $\Delta b_{i}[\%]$ & $\Delta E_{\text {rel }}[\mathrm{eV}]$ & $E_{\text {imp }}[\mathrm{eV}]$ & $\Delta E_{\text {imp }}[\mathrm{eV}]$ \\
\hline \multicolumn{5}{|c|}{$\mathrm{GaN}$} \\
\hline $\mathrm{C}: \mathrm{Gá}$ & -18.1 & 1.65 & e.m.t. & 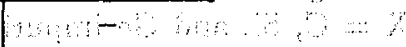 \\
\hline $\mathrm{Si}: \mathrm{Ga}$ & -5.6 & 0.65 & e.m. & $1-1,100$ \\
\hline $\mathrm{Ge}: \mathrm{Ga}$ & $-1: 4$ & 0.25 & e.m. & 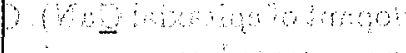 \\
\hline $\mathrm{C}: \mathrm{N}$ & -2.0 & 0.1 & $E_{v}+0.2$ & \\
\hline $\mathrm{Si}: \mathrm{N}$ & 13.6 & 3.9 & $E_{\mathrm{v}}+1.2$ & 0.8 \\
\hline $\mathrm{Ge}: \mathrm{N}$ & 13.5 & 4.1 & $E_{\mathrm{v}}+1.35$ & 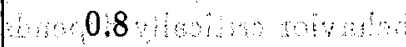 \\
\hline \multicolumn{5}{|c|}{ 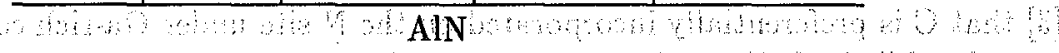 } \\
\hline $\mathrm{C}: \mathrm{Al}$ & -17.2 & 2.6 & $E_{\mathrm{c}}-0.4$ & 1.2 \\
\hline Si:Al & -7.0 & 0.7 & e.m. & \\
\hline $\mathrm{Ge}: \mathrm{Al}$ & -2.9 & 0.3 & e.m & (3) \\
\hline $\mathrm{C}: \mathrm{N}$ & 2.0 & 0.4 & $E_{\mathrm{v}}+0: 4$ & Go 0.1 morom \\
\hline $\mathrm{Si}: \mathrm{N}$ & 16.5 & 6.75 & $E_{\mathrm{v}}+1.7$ & 201001001 \\
\hline Ge:N & 16.7 & 6.95 & $E_{\mathrm{v}}+1.75$ & 1.05 \\
\hline
\end{tabular}

As follows from Table, $\mathrm{C}$; $\mathrm{Si}$, and Ge occupying the cation site in both $\mathrm{GaN}$ and AlN are effective-mass donors. The only exception is $C_{A l}$ in $A l N$, where the 
C-induced level is at about $0.4 \mathrm{eV}$ below the bottom of the conduction band. In $\mathrm{GaN}$, because of its lower band gap, the $\mathrm{C}$-derived level is a resonance situated at $0.9 \mathrm{eV}$ above the bottom of the conduction band.

In addition to the substitutional configuration of $\mathrm{X}_{\text {cation }}$ donors, we have investigated the stability of the DX-like configuration [9]. In this configuration one bond between the impurity and its first neighbor is broken, and one of these atoms (or both) are shifted to an interstitial site. We have analyzed only one possible DX state, namely that with the broken bond between the impurity and the type-1 neighbor; the configuration with the broken bond to the type-2 $\mathrm{N}$ atom should have very similar properties.

We first consider $\mathrm{GaN}$. We find that $\mathrm{C}_{\mathrm{Ga}}^{*}$ is metastable not only in the negative but also in the neutral charge state. The energy of $\mathrm{C}_{\mathrm{Ga}}^{*}$ is higher than that of the substitutional $\mathrm{C}_{\mathrm{Ga}}$ by 0.54 and $0.35 \mathrm{eV}$ for the neutral and the negative charge state, respectively. In the DX state both the host $\mathrm{N}$ atom and the impurity are significantly displaced along the $c$-axis (see Ref. [4] for details). In contrast, $\mathrm{Si}$ is unstable in the DX state, since there is no energy barrier for the transition from the initial DX configuration to the substitutional one. This holds for both the neutral and the negatively charged Si. Finally, a DX state is metastable for the negatively charged $\mathrm{Ge}$, and its energy is higher by $0.3 \mathrm{eV}$ than that of $\mathrm{Ge}_{\mathrm{Ga}}^{-}$. Unlike for $\mathrm{C}_{\mathrm{Ga}}^{*}, \mathrm{Ge}$ atom is located at the lattice site, and the nearest-neighbor $\mathrm{N}$ atom is displaced along the $c$-axis. The $\mathrm{Ge}-\mathrm{N}$ distance is $2.61 \AA$, to be compared with $1.93 \AA$ for the substitutional configuration. $\mathrm{Ge}_{\mathrm{Ga}}^{*}(-)$ introduces a singlet at about $0.4 \mathrm{eV}$ below the bottom of the conduction band. For the neutral charge state, the DX configuration is unstable.

Turning to AlN, we find that in the negative charge state $\mathrm{C}_{\mathrm{Al}}^{*}$ is more stable than $\mathrm{C}_{\mathrm{Al}}^{-1}$ by $0.2 \mathrm{eV}$. In the neutral charge state $\mathrm{C}_{\mathrm{Al}}^{*}$ is metastable, as its energy is higher by $0.48 \mathrm{eV}$ than that of the ground state. Unlike in GaN, the DX configurations are stable for both $\mathrm{Si}$ and $\mathrm{Ge}$ in the negative charge state. Their energies are lower by 0.15 and $1.2 \mathrm{eV}$ than those of the respective substitutional cases. In both cases the impurity remains on the substitutional site, and the $\mathrm{N}$ atom is strongly displaced. For $\mathrm{Si}_{\mathrm{Al}}^{*}$ the distance to the $\mathrm{N}$ atom is $2.95 \AA$ compared to $1.78 \AA$ for $\mathrm{Si}_{\mathrm{Al}}$, and for Ge the respective values are 2.83 and $1.86 \AA$. Si $\mathrm{Sil}_{\mathrm{Al}}^{*}(-)$ introduces a level at $-1.5 \mathrm{eV}$ below the bottom of the conduction band. For Ge, the gap state is even deeper, at $-2.0 \mathrm{eV}$ below the bottom of the conduction band. Finally, the DX configurations are unstable for both $\mathrm{Si}$ and $\mathrm{Ge}$ in the neutral charge state.

The different DX configurations found for $\mathrm{C}$, as opposed to $\mathrm{Si}$ and $\mathrm{Ge}$, are in part due to atomic size effects. For example, when $C$ substitutes the much bigger $\mathrm{Ga}$ atom, the $\mathrm{C}-\mathrm{N}$ bonds are highly stretched, because they are shorter than the equilibrium $\mathrm{Ga}-\mathrm{N}$ bonds by about $15 \%$, In the $\mathrm{DX}$ state one $\mathrm{C}-\mathrm{N}$ bond is broken, and $\mathrm{C}$ is free to relax and to shorten the three remaining, $\mathrm{C}-\mathrm{N}$ bonds. Consequently, the $\mathrm{C}_{\mathrm{Ga}}^{*} \mathrm{~N}$ bonds are shorter by about $7 \%$ than $\mathrm{C}_{\mathrm{Ga}}-\mathrm{N}$ bonds in the substitutional case. At equilibrium, $\mathrm{C}^{*}$ forms a nearly planar configuration with the type-2 neighbors. Such a geometry is additionally stabilized by the tendency of $\mathrm{C}$ to form planar $s p^{2}$ bonds. Both factors may contribute to the stabilization of $\mathrm{C}_{\mathrm{Ga}}^{*}$ in the neutral charge state. In contrast, these factors are not operative for $\mathrm{Si}$ and $\mathrm{Ge}$ impurities, which remain on the substitutional site. 
We shall now consider the acceptors $\mathrm{X}_{\mathrm{N}}$. In zinc-blende crystals, acceptor levels of group-IV atoms are threefold degenerate. Due to the wurtzite symmetry of GaN and AIN, the triplets are split into doublets and singlets by the energy $E_{\text {split }}$. In all cases considered here, the energies of doublets (given in Table), $E_{\mathrm{D}}$, are higher than those of singlets, $E_{\mathrm{S}}$. Both the acceptor energies and the splittings strongly depend on the impurity. We find that $\mathrm{C}$ is a shallow acceptor with $E_{\mathrm{D}}=$ $0.2 \mathrm{eV}$ in $\mathrm{GaN}$, which is in excellent agreement with the recent experimental data [10]. Thus, $\mathrm{C}$ is a promising $p$-type dopant, since it is a shallower acceptor than the commonly used $\mathrm{Mg}$ [2]. In AIN, C is deeper $\left(E_{\mathrm{D}}=0.4 \mathrm{eV}\right)$ and more localized. The doublet-singlet splitting $E_{\text {split }}$ is about $0.2 \mathrm{eV}$ in both materials. In contrast, both $\mathrm{Si}$ and $\mathrm{Ge}$ are deep acceptors. For GaN:Si $E_{\mathrm{D}}=1.2 \mathrm{eV}$ and $E_{\text {split }}=0.6 \mathrm{eV}$, and for GaN:Ge $E_{\mathrm{D}}=1.35 \mathrm{eV}$ and $E_{\text {split }}=0.6 \mathrm{eV}$. In AlN the binding energies and the splittings are even higher, and we find $E_{\mathrm{D}}=1.7(1.75) \mathrm{eV}$ and $E_{\text {split }}=$ $0.7(0.7) \mathrm{eV}$ for $\mathrm{Si}_{\mathrm{N}}$ and $\mathrm{Ge}_{\mathrm{N}}$, respectively.

Finally, we turn to the electronic structure of the nearest-neighbor $\mathrm{X}_{\text {cation }}^{+}-\mathrm{X}_{\mathrm{N}}^{-}$ pairs. Compared to the case of distant $X_{\text {cation }}$ and $X_{N}$ impurities, the main modification is a substantial increase in the doublet-singlet splittings. In the case of $\mathrm{GaN}, E_{\text {split }}$ rises from 0.6 to $1.0 \mathrm{eV}$ for $\mathrm{Si}-\mathrm{Si}$ pairs, and from 0.6 to $1.05 \mathrm{eV}$ for Ge-Ge pairs. For AlN, the increase is from 0.7 to $1.4 \mathrm{eV}$ for Si-Si pairs, and from 0.7 to $1.35 \mathrm{eV}$ for Ge-Ge pairs. This effect is due to the close proximity of the $\mathrm{X}_{\text {cation }}$ donor, located along the $c$-axis relative to the acceptor $\mathrm{X}_{\mathrm{N}}$. The presence of the donor enhances the non-equivalence between the $z$-symmetry singlet and the $(x, y)$-symmetry doublet states.

As it follows from our results, properties of Si and Ge are similar, but they are qualitatively different from those of $\mathrm{C}$. These differences are due to both different atomic energies of their atomic $p$ orbitals and differences in atomic radii, which affect both atomic relaxations and hybridization of the orbitals.

This work was supported in part by grants of the Committee for Scientific Research 2-P03B-178-10, ONR N00014-92-J-1477, and NSF DMR 9408437.

\section{References}

[1] R.F. Davis, Physica $B$ 185, 1 (1993).

[2] H. Morkoc, S. Strite, G.B. Gao, M.E. Lin, B. Sverdlov, M. Burns, J. Appl. Phys. 76, 1363 (1994).

[3] P. Bogusławski, J. Bernholc, to be published.

[4] P. Bogusławski, E.M. Briggs, J. Bernholc, Appl. Phys. Letl., in press.

[5] R. Car, M. Parrinello, Phys. Rev. Lett. 55, 2471 (1985).

[6] P. Bogusławski, E.M. Briggs, J. Bernholc, Phys. Rev. B 51, 17255 (1995).

[7] G. Li, S. Rabii, unpublished (1992).

[8] X. Gonze, R. Stumpf, M. Scheffler, Phys. Rev. B 44, 8503 (1991).

[9] D.J. Chadi, K.J. Chang, Phys. Rev. Lelt. 61, 873 (1988).

[10] S. Fisher, C. Wetzel, E.E. Haller, B.K. Meyer, Appl. Phys. Lett. 67, 1298 (1995). 